\title{
THE ROLE OF IRON IN HEART FAILURE: A LITERATURE REVIEW
}

\author{
Berkin Ersoy (D), Yaren Yamak (D)
}

University of Hamburg School of Medicine, Hamburg, GERMANY

\begin{abstract}
Iron has a key role in the energy generation of the cell. Different organs utilize iron for their diverse needs. The heart is an organ that demands high energy. Nearly one-third of stable heart failure patients and half of the hospitalized patients, regardless of their heart failure status, have anemia. The potential cause of anemia in heart failure patients is found to be multifactorial. Iron deficiency anemia is associated with the reduced number of red blood cells, which transport oxygen into the tissues of the human body. Therefore, in iron deficiency, mitochondria produce less energy. Since iron deficiency has a very important role in the prognosis of heart failure, since the beginning of the 2000s, researchers have conducted several studies and trials, investigating the effect of correcting iron deficiency on the clinical and symptomatic outcomes of heart failure. The most recent results of those studies and trials suggest that intravenous iron therapy in heart failure with reduced ejection fraction patients is associated with improved exercise capability, quality of life, and New York Heart Association class. Ongoing trials such as Ferinject Assessment in Patients with Iron Deficiency and Chronic Heart Failure 2 are aimed more into investigating hospitalization and mortality rates of patients on intravenous iron therapy. Keywords: Iron, anemia, heart failure
\end{abstract}

\section{INTRODUCTION}

According to the study conducted by Shahar et al. (1), heart failure (HF), whether acute or chronic, is known to be one of the most frequent reasons for hospitalizations worldwide, with more than 2000 per 100.000 people aged between 75 to 84 years. Iron deficiency (ID) on the other hand, is a common comorbidity in HF patients, regardless of sex and potential anemia. According to Klip et al. (2), 50\% of the patients with HF suffer from ID. ID causes anomalies in mitochondrial function and cellular energy metabolism and therefore worsens the prognosis of HF patients. Moreover, HF patients show reduced exercise capacity and lower quality of life (QoL) (3). Since the beginning of the 2000s, researchers have conducted several studies and trials, investigating the effect of correcting ID on the clinical and symptomatic outcomes of HF. In this literature review, we illustrate iron metabolism in the human body and its pathophysiology during HF and give a comprehensive overview of the studies and trials investigating the role of iron in HF.

\section{IRON METABOLISM AND ITS PATHOPHYSIOLOGY IN HEART FAILURE}

\section{Iron homeostasis, systemic iron regulation, iron stock}

Iron homeostasis is the resorption, absorption, distribution, and elimination of the biometal iron. It is essential to satisfy the metabolic needs of the body or to carry out specialized functions. Humans have 3.5-5 g iron in their bodies (4). Approximately $65 \%$ of it is allotted to hemoglobin, $10 \%$ is used as functional iron (e.g. myoglobin and ferrous enzymes), and $20 \%$ is used as stocked iron in the body as ferritin (5). Ferritin is mainly stocked in the liver.
Only $0.2 \%$ of the iron in the body is bound to transferrin in circulating form (6).

\section{Iron uptake and absorption}

Cells that need iron express transferrin receptor 1 (TfR-1) and transferrin receptor 2 on their membrane surfaces. Transferrin binds to them and the complex is taken up into the cell by endocytosis and is processed in the lysosomes. From there the iron can be integrated into its target proteins (7).

The resorption rate of iron in the gut depends on the iron demand of the cells. If there is an iron need in the body (e.g. loss of blood), resorption of the iron through diet can increase up to $30 \%$ (8). Another way for the body to produce iron is to destroy the red blood cells and free the iron from hemoglobin (9).

\section{Duodenal iron uptake}

In the stomach, iron is transformed from its ferrous form into the ferric form with the contribution of hydrochloric acid and vitamin $\mathrm{C}$, and then transferred to the duodenum. Like other minerals, iron is absorbed in the duodenum. The amount of iron resorbed in the duodenum is an important factor for systemic iron homeostasis (10). In the duodenum, it is either taken up through the duodenal mucosa transporters such as divalent metal transporter 1 (DMT1) of the enterocytes as $\mathrm{Fe}^{3+}$ (non-heme iron) by symport with the protons or as heme iron over heme carrier protein 1 (11). At the basolateral side of the cell, the iron is then delivered into the bloodstream in reduced form as $\mathrm{Fe}^{2+}$ (heme iron) by the ferroportin of the mucosa cells (7). The $\mathrm{Fe}^{2+}$ is oxidized to $\mathrm{Fe}^{3+}$ and then bound to apo-transferrin (5). The apo-transferrin and ferric iron $\left(\mathrm{Fe}^{3+}\right)$ make up the transferrin. 


\section{Systemic iron homeostasis}

The iron concentration in the organism is regulated by the protein hepcidin, which binds to ferritin in the small intestine and initiates its degradation (12). If the blood iron concentration is too low, the liver decreases hepcidin production. As a result, the release of iron into the bloodstream is increased (13). On the contrary, if the hepatocyte cells are provided with enough iron, they increase the hepcidin production in the liver. Hepcidin also controls the release of iron from the cells of the reticuloendothelial system and macrophages (14).

\section{Cellular iron homeostasis}

The regulation of cellular iron homeostasis depends on whether the cells have an iron deficit or iron repletion (4). On the cellular level, iron homeostasis is regulated by the expression of transferrin receptor (TfR), apo-ferritin, and 5-aminolevulinic acid (ALA) synthase, the key enzyme of the heme biosynthesis (15). Having different stages and levels of manifestation, the best-understood mechanism of iron homeostasis is the iron-dependent binding of iron regulatory proteins (IRPs) (16). In the case of a cell sufficiently provided with iron, the IRPs bind onto the iron-responsive elements (IREs) in the 5' end of the untranslated region of the ferritin messenger ribonucleic acid (mRNA), which has a blocking effect on the molecule, so that the message cannot be translated when there is no need for iron storage. Contemporaneously, the IRPs bind on the 3' uridine triphosphates of the TfR-1 mRNA which ensures the protection of the message from degradation in the cytoplasm, therefore increasing the expression of the receptor on the cell membrane (16). Whereas there is no need for extra iron in the cell, the IRPs do not dock onto IREs, which then allows ferritin translation. This process exposes the TfR-1 mRNA for degradation in the cytoplasm so that the iron uptake can be limited, and cellular iron storage is supported. The messenger ribonucleic acids of TfR, apo-ferritin, DMT-1, ferroportin 1, ALA synthase 2, and hypoxia-inducible factor $2 \alpha$ contain IREs, which makes them important players in cellular iron homeostasis $(16,17)$.

\section{Cellular iron metabolism}

Intracellular iron can be utilized, imported by mitochondria, or bounded to ferritin in the cytoplasm (15). Free iron is harmful to cells since it can catalyze the generation of cytotoxic reactive oxygen species (18). To prevent this from occurring, iron is stored in the cells as ferritin (19).

\section{Iron in mitochondria}

Iron has a key role in the energy generation of the cell, like the enzymes that are responsible for energy production in the citric acid cycle -such as cytochrome oxidase. In mitochondria, iron is used for heme synthesis and the generation of iron-sulfur clusters in the electron transport chain (20).

\section{Heme synthesis}

Organisms produce the tetrapyrrole porphyrin ring of heme from the forerunner ALA, via an eight-step enzymatic pathway (21). ALA is generated in the mitochondria of eukaryotes by a condensation reaction of succinyl-coenzyme A and glycine, catalyzed by ALA synthase (22). The molecule is then exported to the cytosol, where it is enzymatically converted into intermediate mediators. In the final reaction of the heme biosynthesis pathway, ferrochelatase catalyzes the insertion of $\mathrm{Fe}^{2+}$ into the mediator protoporphyrin IX (23). Synthesized heme is then exported to the cytosol to be incorporated into hemoproteins (21).
With that in mind, it is safe to say that iron deficiency can cause interruption of heme synthesis in erythroid cells and cause necrosis both in erythroid cells as well as in non-erythroid cells (24). That may result in microcytic anemia or iron deficiency anemia in general.

\section{Iron regulation in the heart}

Different organs utilize iron for their needs. When it comes to the cardiomyocytes, both iron deficiency and iron overload can cause pathologies in cardiac function. Ferroportin, as discussed previously, plays an essential role in iron uptake at the systemic level. However, it is also proven to be found in other cell tissues with no known contribution to systemic iron regulation (25).

\section{Iron and cardiovascular disease - pathology relations}

The heart is an organ with high energy demand. Iron deficiency is found in $30-50 \%$ of patients with HF thereby causes severe symptoms and poor prognosis $(2,26)$.

It remains unclear, whether iron also plays a role in atherosclerosis. Potential disease mechanisms might be iron-driven endothelial cell dysfunction, oxidative stress, monocyte adhesion, and plaque instability (27). However, experimental data argue against any significant pathologic relation of macrophage iron in atherosclerotic changes (28).

\section{Anemia and heart failure}

Anemia is a frequent comorbidity in patients with HF and affects nearly one-third of the stable patients and one-half of the hospitalized patients, irrespective of the HF subtype (29-33).

Compared to non-anemic patients with HF, anemic HF patients are associated with increased age and clinical manifestations of diabetes and chronic kidney disease (CKD). Anemic HF patients are also found to have increased blood pressure, increased levels of neurohormones, proinflammatory cytokines, and the use of diuretics. Moreover, those patients are expected to have lower QoL and exercise capacity, which is determined by the 6-minute walking time. All these findings suggest that anemia is a chronic disease that can develop with symptoms such as low levels of bone marrow function, decreased erythropoietin synthesis, and insufficient iron utilization (34).

The potential cause of anemia in HF patients is found to be multifactorial. Reduced hematinic vitamins such as vitamin B12 and folic acid can be seen in a minority of HF patients (35).

Levels of erythropoietin are reduced in HF. Erythropoietin is produced primarily in the kidneys, precisely within the renal cortex and outer medulla by peritubular fibroblasts. In patients with HF, renal blood flow (RBF) is reduced, which can increase the production of erythropoietin due to low partial pressure of oxygen, which activates the inducer of the erythropoietin gene transcription, hypoxia-inducible factor- $1 \alpha$ (36). The increase of erythropoietin levels correlates with the severity of HF (37).

Angiotensin receptor blockers and angiotensin-converting enzyme inhibitors are commonly used drugs in HF treatment. Angiotensin II (AII) reduces RBF and increases oxygen demand, which stimulates erythropoietin production. Secondly, AII stimulates bone marrow progenitor cell production directly. As both of the previously named drugs inhibit the availability of AII, they cause a modest reduction in hemoglobin by reducing the production of erythropoietin and erythroid progenitors, as well as by blocking the disintegration of the hematopoiesis inhibitor $\mathrm{N}$-acetyl-seryl-aspartyl-lysyl-proline (38).

Androne et al. (39) reported that nearly $50 \%$ of the patients introduced for cardiac transplant had hemodilution-induced pseudo- 
anemia, despite having normal plasma volume. Hemoglobin analysis shows true anemia, determined by the volume of red blood cells, in the majority of the anemic HF patients (40).

Another major factor for anemia in HF patients is the proinflammatory state they are found to be in. As discussed before, proinflammatory cytokines such as but not limited to tumor necrosis factor- $\alpha$ (TNF- $\alpha$ ), interleukin-6 (IL-6), and C-reactive protein are increased in HF. IL- 6 and TNF- $\alpha$ activate GATA binding factor 2 (a protein that binds GATA sequence in target gene promoters) and nuclear factor- $\kappa \mathrm{B}$, which act as inhibitors of erythropoietin production (41). Furthermore, IL-6 stimulates hepatocytes for increasing hepcidin synthesis and as a result, blocks duodenal iron uptake (42). By the same token, IL-6 reduces the expression of ferroportin, and therefore, iron release into the bloodstream is prevented (42).

As HF can cause anemia, it appears that the mechanism also works the other way around. In another study, when hemoglobin was elevated up to $5.5 \mathrm{~g} / \mathrm{dL}$ with erythropoietin in patients with moderate anemia and $\mathrm{CKD}$, cardiac output, and left ventricular ejection fraction (LVEF) shortening decreased progressively and anti-proportionally to the hemoglobin increase (43). Thus, the evidence argues that increasing hemoglobin levels in heart failure with reduced ejection fraction (HFrEF) patients can be associated with reduced LVEF, boosted left ventricle afterload, and extended vascular resistance, which would explain the inverse association of hemoglobin and $\operatorname{LVEF}(31,44,45)$.

A meta-analysis of 33 studies, involving more than 150.000 patients with HF, showed that anemia increased the relative risk of death by $100 \%$ (46). On the other hand, it is unlikely that there is a linear association between hemoglobin level and mortality. A majority of the increased risk arises at low hemoglobin $(47,48)$. A J-shaped relation between hemoglobin and mortality rate has been shown in the normal population, as well as in patients with acute coronary syndrome, coronary heart disease, and HF (49-51). The hemoglobin range of 13 to $16 \mathrm{~g} / \mathrm{dL}$ provides the lowest mortality risk, with increased risk below or above this interval.

As already discussed, the pathomechanism of anemia as a comorbidity in HF patients is multifunctional. The hemodynamic, renal, and neurohormonal alterations are triggered when the oxygen delivered to metabolizing tissues is decreased. This leads to elevated workload in myocardial cells, which later on may cause diverse left ventricular remodeling and hypertrophy of the myocardium $(30,52)$.

Within 148 patients with stable chronic HF, Opasich et al. (31) determined a specific cause of anemia in $43 \%$, an iron deficiency in $5 \%$, and insufficient erythropoietin production, proinflammatory cytokine activation, or defective iron utilization notwithstanding adequate iron storage indicating anemia of chronic disease with functional iron deficiency in $57 \%$ of all patients. Regardless of the anemic status, iron deficiency is a comorbidity in HF, which cannot be ignored. Iron deficiency anemia results in a decreased number of red blood cells (24). Therefore, one may tire easier than normal and show poor resilience.

According to previous studies HF patients are often iron deficient. Current guidelines of the European Society of Cardiology recommend iron supplementation in HFrEF patients for the improvement of symptoms (53). Clinical trials of intravenous iron supplementation in HF patients usually define ID as having a ferritin level between 100-300 $\mu \mathrm{g} / \mathrm{L}$ with transferrin saturation (TSAT) $<20 \%$ or ferritin level less than $100 \mu \mathrm{g} / \mathrm{L}$, and these trials have demonstrated improvement in symptoms and functional outcomes (54).

\section{OVERVIEW OF CLINICAL TRIALS}

\section{Therapy with erythropoiesis-stimulating agents}

The Reduction of Events with Darbepoetin alfa in Heart Failure (RED-HF) trial was conducted to investigate erythropoiesis-stimulating agents' (ESAs) usage on anemic patients with HFrEF. The study was published in 2013 (55). Previous to the RED-HF trial, 13 smaller studies investigated the potential benefits of ESAs. As reported in the meta-analysis by Kotecha et al. (56), ESAs led to improvement of symptoms, improved 6-minute walk distance (6MWD), improvement in New York Heart Association (NYHA) class, QoL, and an increase in hemoglobin by $2 \mathrm{~g} / \mathrm{dL}$. Furthermore, erythropoiesis-stimulating agent therapy was shown to reduce HF-related hospitalization (56). However, the RED-HF trial, being a pivotal double-blind placebo-controlled trial, did not support the promising results of these 13 small studies. In the RED-HF trial, the non-placebo group received darbepoetin alpha to increase and maintain the hemoglobin level of $13 \mathrm{~g} / \mathrm{dL}$ (57). The trial had a median follow-up time of 28 months and thereafter darbepoetin usage resulted in no benefits concerning primary outcomes, such as mortality rate, from any cause, and hospitalization for worsening HF. Compared to the placebo group, darbepoetin increased the hemoglobin level by around $1.5 \mathrm{~g} / \mathrm{dL}$ throughout the trial and therefore a marginal improvement of QoL was seen in the darbepoetin alfa group. On the other hand, in the darbepoetin alfa group, a statistically significant increase in the occurrence of thrombotic and embolic events was seen $(\mathrm{p}=0.01)(57)$.

\section{Oral iron therapy}

Since the RED-HF trial demonstrated that treating anemia by increasing hemoglobin levels in HFrEF patients did not significantly improve the prognosis of HF, the focus shifted to replacing the deficient iron. The oral iron replacement was first investigated in a study by Silverberg et al. (58). Iron Repletion Effects on Oxygen Uptake in Heart Failure trial, being a phase 2 double-blind randomized controlled trial, is the biggest study, which investigated the outcomes of oral iron preparations on HF patients. Within the trial, the non-placebo group received oral iron polysaccharide $150 \mathrm{mg}$ twice daily (59). After 16 weeks of follow-up in the non-placebo group, there were no statistically significant changes neither in the primary endpoint (peak VO2) nor in the secondary endpoints (6MWD, QoL, etc.) (60). These findings were not coherent with the findings of intravenous iron trials on similar populations (61). The reason for non-beneficial outcomes of oral iron preparations is suspected to be elevated hepcidin levels of patients with HF, which then inhibits the absorption of iron in enterocytes by reducing transmembrane ferroportin. As mentioned above, reduced transmembrane ferroportin limits the transport of iron from enterocytes to blood (3).

\section{Intravenous iron therapy}

Ferinject Assessment in Patients with Iron Deficiency and Chronic Heart Failure (FAIR-HF) trial was the first large trial in which the outcomes of intravenous iron preparations on patients with HFrEF were investigated. The trial was conducted between 2007 and 2008 and the results were published in 2009 (62). A total of 459 patients with NYHA class II or III, LVEF less than $45 \%$, and ID (ferritin level between 100-300 $\mu \mathrm{g} / \mathrm{L}$ with TSAT $<20 \%$ or ferritin less than $100 \mu \mathrm{g} / \mathrm{L}$ ) were enrolled into the study and randomized 2:1 into the non-placebo and placebo groups (63). In the correction phase, the non-placebo group received weekly ferric carboxy-malt- 
ose (FCM), the equivalent of $200 \mathrm{mg}$ of iron. In the maintenance phase ( 8 or 12 weeks after the beginning), the frequency was reduced to every 4 weeks with the same amount of FCM until the end of the study (63). In terms of primary endpoints, at week 24, one-half of the patients in the FCM group were found to be much or moderately improved according to the Patient Global Assessment (PGA), whereas only $28 \%$ of the patients in the placebo group found to be as such. There was a reduction in NYHA class in the FCM group when compared to the placebo group, which was also statistically significant $(\mathrm{p}<0.001)$. The improvements of the FCM group were regardless of potential anemia (62).

The Ferric Carboxymaltose Evaluation on Performance in patients with Iron Deficiency in Combination with Chronic Heart Failure (CONFIRM-HF) trial was conducted between 2011 and 2013 and the results were published in 2015 (64). The design of the trial showed similarities to FAIR-HF, but the FCM doses were higher (the equivalent of 500-1000 $\mathrm{mg}$ of iron), therapy duration (52 weeks) was longer, and randomization was done in a 1:1 ratio in 304 participating patients (65). In terms of the primary endpoint of the CONFIRM-HF, patients in the FCM group at week 24 and onwards had an improved 6MWD, when compared to the placebo group ( $p>0.01)$. There were also improvements in the secondary endpoints (NYHA class, QoL, PGA) (64). Furthermore, CONFIRM-HF indicated that FCM therapy might cause a reduction in the risk of hospitalization due to worsening HF. This finding has once again shifted the focus from symptomatic improvement to clinical improvements such as but not limited to hospitalization for any reason or due to cardiovascular events and mortality rate.

After CONFIRM-HF, in 2017, the results of the Effect of Ferric Carboxymaltose on Exercise Capacity in Patients With Iron Deficiency and Chronic Heart Failure trial were published (66). The inclusion criteria were nearly identical when compared with other past trials, additionally including hemoglobin levels lower than $15 \mathrm{~g} / \mathrm{dL}$, brain natriuretic peptide greater than $100 \mathrm{pg} / \mathrm{mL}$ or N-terminal-pro hormone brain natriuretic peptide greater than $400 \mathrm{pg} /$ $\mathrm{mL}$, and peak VO2 of 10 to $20 \mathrm{~mL} . \mathrm{kg}-1 . \mathrm{min}-1.172$ patients were enrolled in the trial and were randomized in 1:1 ratio into FCM and placebo groups. The FCM group received the same dose (the equivalent of 500-1000 mg of iron) as in the CONFIRM-HF trial for 24 weeks. The primary endpoint changes in peak VO2 levels were investigated, which showed no significant improvement in the FCM group. In terms of secondary endpoints, PGA and NYHA class, there were significant improvements in the FCM group $(\mathrm{p}<0.05$ for both results) (66).

Most recently in late 2020, the results of a Randomized, Double-Blind Placebo-Controlled Trial Comparing the Effect of Intravenous Ferric Carboxymaltose on Hospitalizations and Mortality in Iron Deficient Subjects Admitted for Acute Heart Failure (AFFIRM-AHF) trial were published (67). The trial had the highest sample size $(n=1132)$ compared to the other FCM in HF trials. In this particular trial, eligible patients were hospitalized for acute $\mathrm{HF}$ with accompanying ID (ferritin levels lower than $100 \mu \mathrm{g} / \mathrm{L}$, or between 100-299 $\mu \mathrm{g} / \mathrm{L}$ with transferrin saturation <20\%) and LVEF of less than $50 \%$. Patients were randomized before their hospital discharge 1:1 into FCM or placebo groups. The FCM group was given intravenous FCM according to their weight and ID status for 24 weeks, which was equivalent to $500-1000 \mathrm{mg}$ of iron in each dose (68). The patients were also followed up after their hospital discharge with no longer than 6 week-periods. The AFFIRM-AHF trial used the rate of recurrent HF hospitalizations and cardiovascular mortality up to 52 weeks after randomization as its primary outcomes. During the follow-ups for 52 weeks, a total of 293 (52.5\%) primary outcome events occurred in the FCM group, whereas 372
(67.6\%) events were recorded in the placebo group (67). Although this was only borderline significant $(\mathrm{p}=0.059)$, the findings are very promising for the FCM therapy, since the trial showed that fewer days were lost due to HF hospitalization in the FCM group (369 days per 100 patient-years) compared to the placebo group (548 days per 100 patient-years). Furthermore, there was no significant difference between the two groups about cardiovascular death (67).

As the trials were conducted, meta-analyses using individual patient data of several FCM in HF trials were reported. Anker et al. (61) published a meta-analysis in 2018, which was based on 4 randomized control trials (RCTs) (FER-CARS-01, FAIR-HF, EFFICACY-HF, and CONFIRM-HF). In their meta-analysis, they focused on the clinical outcomes such as but not limited to recurrent cardiovascular hospitalizations and cardiovascular death. The data of a total of 839 patients were examined. When compared with the placebo groups, patients in FCM groups had a lower rate of recurrent cardiovascular or HF hospitalization, and cardiovascular mortality. Moreover, there was no increase in adverse events associated with the FCM groups (61). In a recent meta-analysis by Khan et al. (69), the data of AFFIRM-AHF was examined along with the 4 RCTs included in the meta-analysis of Anker et al. (61). Khan et al. (69) included a total of 1947 patients from 5 RCTs and the outcomes of interests were HF hospitalizations, all-cause mortality, and cardiovascular mortality. The findings of this meta-analysis showed that FCM therapy, when compared to the placebo groups, statistically significantly reduced the time to first heart failure hospitalization or death for cardiovascular causes (hazard ratio $=0.76$; $95 \%$ confidence interval $(\mathrm{CI})=0.63-0.90 ; \mathrm{I} 2=555)$. In addition, FCM therapy was indicated to reduce the risk of recurrent $\mathrm{HF}$ and cardiovascular hospitalization. On the other hand, there was no significant reduction in all-cause (odds ratio $(\mathrm{OR})=0.97 ; 95 \% \mathrm{CI}=0.73-1.28$; $\mathrm{I} 2=0 \%)$ or cardiovascular $(\mathrm{OR}=0.93 ; 95 \% \mathrm{CI}=0.69-1.27 ; \mathrm{I} 2=0 \%)$ mortality rates (69).

The currently ongoing trial Ferinject Assessment in Patients with Iron Deficiency and Chronic Heart Failure 2 intends to investigate the effect of intravenous FCM in HFrEF patients, taking the rate of recurrent hospitalizations due to heart failure and cardiovascular death during follow-up as its primary endpoints. The trial is planned to have a total of circa 200 patients, who had present chronic HF for at least 12 months from the initial diagnosis (70).

\section{CONCLUSION}

With iron being a crucial biometal complex in homeostasis, iron deficiency is a common finding in HF patients regardless of their anemia status. HF patients with ID show more severe symptoms and have an impaired prognosis. Throughout the last 20 years, several studies evaluated the role of iron supplementation in HF. They demonstrated that intravenous iron therapy in HF patients is associated with improved exercise capability, QoL, and NYHA class. The beneficial effects of intravenous iron therapy on hospitalization and mortality rate are also shown partially in several trials, but further trials and studies are needed to have a better understanding of this aspect of the topic.

\section{Ethics Committee Approval: N/A \\ Informed Consent: N/A \\ Conflict of Interest: The authors declared no conflict of interest.}

Author Contributions: Concept: BE, YY. Design: BE, YY. Supervision: BE, YY. Resources: BE, YY. Materials: BE, YY. Data collection and/or processing BE, YY. Analysis and/or Interpretation: BE, YY. Literature Search: BE, YY. Writing Manuscript: BE, YY. Critical Review: BE, YY. 
Financial Disclosure: The authors declared that this study received no financial support.

Editor-in-Chief's Note: One author of this article, Berkin Ersoy is a member of the editorial board of Turkish Medical Student Journal. However, he did not take place in any stage of the editorial decision of the manuscript. The editors who evaluated this manuscript are from other institutions.

\section{REFERENCES}

1. Shahar E, Lee S, Kim J et al. Hospitalized heart failure: rates and long-term mortality. J Card Fail 2004;10(5):374-9.

2. Klip IT, Comin-Colet J, Voors AA et al. Iron deficiency in chronic heart failure: an international pooled analysis. Am Heart J 2013;165(4):575-82.e3.

3. Anand IS, Gupta P. Anemia and iron deficiency in heart failure: current concepts and emerging therapies. Circulation 2018;138(1):80-98

4. Anderson GJ, Frazer DM. Current understanding of iron homeostasis. Am J Clin Nutr 2017;106(6):1559-66.

5. Wallace DF. The regulation of iron absorption and homeostasis. Clin Biochem Rev 2016;37(2):51-62

6. Saito H. Metabolism of iron stores. Nagoya J Med Sci 2014;76(3-4):235-54.

7. Umbreit JN, Conrad ME, Moore EG et al. Iron absorption and cellular transport: the mobilferrin/paraferritin paradigm. Semin Hematol 1998;35(1):13-26.

8. Beck KL. Anemia: prevention and dietary strategies. In: Caballero B, editor. Encyclopedia of Food and Health. Oxford: Academic Press; 2016.p.164-8.

9. Maitra D, Byun J, Andreana PR et al. Reaction of hemoglobin with $\mathrm{HOCl}$ mechanism of heme destruction and free iron release. Free Radic Biol Med 2011;51(2):374-86.

10. Enculescu M, Metzendorf C, Sparla R et al. Modelling systemic iron regulation during dietary iron overload and acute inflammation: role of hepcidin-independent mechanisms. PLoS Comput Biol 2017;13(1):e1005322.

11. Rassow J, Hauser K, Netzker R et al. Duale Reihe Biochemie. Thieme; 2016.p.324.

12. Gulec S, Anderson GJ, Collins JF. Mechanistic and regulatory aspects of intestinal iron absorption. Am J Physiol Gastrointest Liver Physiol 2014;307(4):397-409.

13. Loreal O, Cavey T, Bardou-Jacquet E et al. Iron, hepcidin, and the metal connection. Front Pharmacol 2014;5:128.

14. Ganz T. Systemic iron homeostasis. Physiol Rev 2013;93(4):1721-41.

15. MacKenzie EL, Iwasaki K, Tsuji Y. Intracellular iron transport and storage: from molecular mechanisms to health implications. Antioxid Redox Signal 2008;10(6):997-1030.

16. Wilkinson N, Pantopoulos K. The IRP/IRE system in vivo: insights from mouse models. Front Pharmacol 2014;5:176.

17. Mastrogiannaki M, Matak P, Peyssonnaux C. The gut in iron homeostasis: role of HIF-2 under normal and pathological conditions. Blood 2013;122(6):885-92.

18. Dixon SJ, Stockwell BR. The role of iron and reactive oxygen species in cell death. Nat Chem Biol 2014;10(1):9-17.

19. Orino K, Lehman L, Tsuji $Y$ et al. Ferritin and the response to oxidative stress. Biochem J 2001;357(1):241-7.

20. Ponka P. Tissue-specific regulation of iron metabolism and heme synthesis: distinct control mechanisms in erythroid cells. Blood 1997;89(1):1-25.

21. Severance S, Hamza I. Trafficking of heme and porphyrins in metazoa. Chem Rev 2009;109(10):4596-616.

22. Kumari A. Heme synthesis. In: Kumari A, editor. Sweet Biochemistry: Academic Press; 2018.p.33-6.

23. Ferreira GC, Franco R, Lloyd SG et al. Structure and function of ferrochelatase. J Bioenerg Biomembr 1995;27(2):221-9.

24. Haq RU, Wereley JP, Chitambar CR. Induction of apoptosis by iron deprivation in human leukemic CCRF-CEM cells. Exp Hematol 1995;23(5):428-32.

25. Ghafourian K, Shapiro JS, Goodman L et al. Iron and heart failure: diagnosis, therapies, and future directions. JACC Basic Transl Sci 2020;5(3):300-13.

26. Comin-Colet J, Enjuanes C, Gonzalez G et al. Iron deficiency is a key determinant of health-related quality of life in patients with chronic heart failure regardless of anaemia status. Eur J Heart Fail 2013;15(10):1164-72.

27. Kuo KL, Hung SC, Lee TS et al. Iron sucrose accelerates early atherogenesis by increasing superoxide production and upregulating adhesion molecules in CKD. J Am Soc Nephrol 2014;25(11):2596-606.

28. Kautz L, Gabayan V, Wang X et al. Testing the iron hypothesis in a mouse model of atherosclerosis. Cell Rep 2013;5(5):1436-42.

29. Anand IS, Kuskowski MA, Rector TS et al. Anemia and change in hemoglobin over time related to mortality and morbidity in patients with chronic heart failure: results from Val-HeFT. Circulation 2005;112(8):1121-7.

30. Anand I, McMurray JJ, Whitmore J et al. Anemia and its relationship to clinical outcome in heart failure. Circulation 2004;110(2):149-54

31. Opasich C, Cazzola M, Scelsi L et al. Blunted erythropoietin production and defective iron supply for erythropoiesis as major causes of anaemia in patients with chronic heart failure. Eur Heart J 2005;26(21):2232-7.

32. Al-Ahmad A, Rand WM, Manjunath G et al. Reduced kidney function and anemia as risk factors for mortality in patients with left ventricular dysfunction. J Am Coll Cardiol 2001;38(4):955-62.
33. Horwich TB, Fonarow GC, Hamilton MA et al. Anemia is associated with worse symptoms, greater impairment in functional capacity and a significant increase in mortality in patients with advanced heart failure. J Am Coll Cardiol 2002;39(11):1780-6.

34. Anand IS. Anemia and chronic heart failure. Journal of the American College of Cardiology 2008;52(7):501-11.

35. Witte KK, Desilva R, Chattopadhyay S et al. Are hematinic deficiencies the cause of anemia in chronic heart failure? Am Heart J 2004;147(5):924-30.

36. Bauer C, Kurtz A. Oxygen sensing in the kidney and its relation to erythropoietin production. Annu Rev Physiol 1989;51:845-56.

37. Volpe M, Tritto C, Testa U et al. Blood levels of erythropoietin in congestive heart failure and correlation with clinical, hemodynamic, and hormonal profiles. Am J Cardiol 1994;74(5):468-73.

38. van der Meer P, Lipsic E, Westenbrink BD et al. Levels of hematopoiesis inhibitor $\mathrm{N}$-acetyl-seryl-aspartyl-lysyl-proline partially explain the occurrence of anemia in heart failure. Circulation 2005;112(12):1743-7.

39. Androne AS, Katz SD, Lund L et al. Hemodilution is common in patients with advanced heart failure. Circulation 2003;107(2):226-9.

40. Montero D, Lundby C, Ruschitzka F et al. True anemia-red blood cell volume deficit-in heart failure: a systematic review. Circ Heart Fail 2017;10(5):e003610.

41. Jelkmann W. Proinflammatory cytokines lowering erythropoietin production. J Interferon Cytokine Res 1998;18(8):555-9.

42. Weiss G, Goodnough LT. Anemia of chronic disease. N Engl J Med 2005;352(10):1011-23.

43. McMahon LP, Mason K, Skinner SL et al. Effects of haemoglobin normalization on quality of life and cardiovascular parameters in end-stage renal failure. Nephrol Dial Transplant 2000;15(9):1425-30.

44. O'Meara E, Clayton T, McEntegart MB et al. Clinical correlates and consequences of anemia in a broad spectrum of patients with heart failure: results of the Candesartan in Heart Failure: Assessment of Reduction in Mortality and Morbidity (CHARM) Program. Circulation 2006;113(7):986-94.

45. Ezekowitz JA, McAlister FA, Armstrong PW. Anemia is common in heart failure and is associated with poor outcomes: insights from a cohort of 12065 patients with new-onset heart failure. Circulation 2003;107(2):223-5.

46. Groenveld HF, Januzzi JL, Damman $\mathrm{K}$ et al. Anemia and mortality in heart failure patients a systematic review and meta-analysis. J Am Coll Cardiol 2008;52(10):818-27.

47. Komajda M, Anker SD, Charlesworth A et al. The impact of new onset anaemia on morbidity and mortality in chronic heart failure: results from COMET. Eur Heart J 2006;27(12):1440-6.

48. Sharma R, Francis DP, Pitt B et al. Haemoglobin predicts survival in patients with chronic heart failure: a substudy of the ELITE II trial. Eur Heart J 2004;25(12):1021-8.

49. Gagnon DR, Zhang TJ, Brand FN et al. Hematocrit and the risk of cardiovascular disease--the Framingham study: a 34-year follow-up. Am Heart J 1994;127(3):674-82.

50. Sabatine MS, Morrow DA, Giugliano RP et al. Association of hemoglobin levels with clinical outcomes in acute coronary syndromes. Circulation 2005;111(16):2042-9.

51. Brown DW, Giles WH, Croft JB. Hematocrit and the risk of coronary heart disease mortality. Am Heart J 2001;142(4):657-63.

52. Datta BN, Silver MD. Cardiomegaly in chronic anaemia in rats; gross and histologic features. Indian J Med Res 1976;64(3):447-58.

53. Ponikowski P, Voors AA, Anker SD et al. 2016 ESC Guidelines for the diagnosis and treatment of acute and chronic heart failure: The Task Force for the diagnosis and treatment of acute and chronic heart failure of the European Society of Cardiology (ESC) developed with the special contribution of the Heart Failure Association (HFA) of the ESC. European Heart Journal 2016;37(27):2129-200.

54. Grote Beverborg N, Klip IT, Meijers WC et al. Definition of iron deficiency based on the gold standard of bone marrow iron staining in heart failure patients. Circ Heart Fail 2018;11(2):e004519.

55. Swedberg K, Young JB, Anand IS et al. Treatment of anemia with darbepoetin alfa in systolic heart failure. N Engl J Med 2013;368(13):1210-9.

56. Kotecha D, Ngo K, Walters JA et al. Erythropoietin as a treatment of anemia in heart failure: systematic review of randomized trials. Am Heart J 2011;161(5):82231.e2.

57. McMurray JJ, Anand IS, Diaz R et al. Baseline characteristics of patients in the Reduction of Events with Darbepoetin alfa in Heart Failure trial (RED-HF). Eur J Heart Fail 2013;15(3):334-41

58. Silverberg DS, Wexler D, Blum $\mathrm{M}$ et al. The use of subcutaneous erythropoietin and intravenous iron for the treatment of the anemia of severe, resistant congestive heart failure improves cardiac and renal function and functional cardiac class, and markedly reduces hospitalizations. J Am Coll Cardiol 2000;35(7):1737-44.

59. Lewis GD, Semigran MJ, Givertz MM et al. Oral iron therapy for heart failure with reduced ejection fraction: design and rationale for oral iron repletion effects on oxygen uptake in heart failure. Circ Heart Fail 2016;9(5):e000345.

60. Lewis GD, Malhotra R, Hernandez AF et al. Effect of oral iron repletion on exercise capacity in patients with heart failure with reduced ejection fraction and iron deficiency: the IRONOUT HF randomized clinical trial. JAMA 2017;317(19):1958-66. 
61. Anker SD, Kirwan BA, van Veldhuisen DJ et al. Effects of ferric carboxymaltose on hospitalisations and mortality rates in iron-deficient heart failure patients: an individual patient data meta-analysis. Eur J Heart Fail 2018;20(1):125-33.

62. Anker SD, Comin-Colet J, Filippatos G et al. Ferric carboxymaltose in patients with heart failure and iron deficiency. N Engl J Med 2009;361(25):2436-48.

63. Anker SD, Comin-Colet J, Filippatos G et al. Rationale and design of Ferinject assessment in patients with IRon deficiency and chronic Heart Failure (FAIR-HF) study: a randomized, placebo-controlled study of intravenous iron supplementation in patients with and without anaemia. Eur J Heart Fail 2009;11(11):1084-91.

64. Ponikowski P, van Veldhuisen DJ, Comin-Colet J et al. Beneficial effects of longterm intravenous iron therapy with ferric carboxymaltose in patients with symptomatic heart failure and iron deficiencydagger. Eur Heart J 2015;36(11):657-68.

65. Ponikowski P, van Veldhuisen DJ, Comin-Colet J et al. Rationale and design of the CONFIRM-HF study: a double-blind, randomized, placebo-controlled study to assess the effects of intravenous ferric carboxymaltose on functional capacity in patients with chronic heart failure and iron deficiency. ESC Heart Fail 2014;1(1):52-8.

66. van Veldhuisen DJ, Ponikowski P, van der Meer P et al. Effect of ferric carboxymaltose on exercise capacity in patients with chronic heart failure and iron deficiency. Circulation 2017;136(15):1374-83.
67. Ponikowski P, Kirwan B-A, Anker SD et al. Ferric carboxymaltose for iron deficiency at discharge after acute heart failure: a multicentre, double-blind, randomised, controlled trial. The Lancet 2020;396(10266):1895-904.

68. Ponikowski P, Kirwan BA, Anker SD et al. Rationale and design of the AFFIRM-AHF trial: a randomised, double-blind, placebo-controlled trial comparing the effect of intravenous ferric carboxymaltose on hospitalisations and mortality in iron-deficient patients admitted for acute heart failure. Eur J Heart Fail 2019;21(12):1651-8.

69. Khan MS, Usman MS, von Haehling S et al. Ferric carboxymaltose for the treatment of iron-deficient heart failure patients: a systematic review and meta-analysis. ESC Heart Fail 2020;7(6):3392-400.

70. Intravenous Iron in Patients with Systolic Heart Failure and Iron Deficiency to Improve Morbidity \& Mortality (FAIR-HF2) (serial online) (cited 2021 May 24) Available from: URL:https://clinicaltrials.gov/ct2/show/NCT03036462?term=FAIR-HF2\&draw=2\&rank=1. 